\title{
Thorakale und abdominale Organverletzungen bei Kindern und Jugendlichen
}

Peter P. Schmittenbecher

\section{Einleitung}

Unfälle sind die häufigste Todesursache im Kindesalter. Während bei den Kleinkindern Stürze im Haushalt, im Kindergarten und auf dem Spielplatz im Vordergrund stehen, nimmt mit zunehmendem Alter der Anteil von Sport- und Verkehrsunfällen zu. Insassen- und Mitfahrerunfälle werden in der Adoleszenz zunehmend von Fahrerunfällen (Fahrrad, Moped) abgelöst. Im Jahr 2012 wurden in der BRD 308 letale Kinderunfälle < 15 Jahre registriert.

Thoraxtraumen sind in 5,4\% beteiligt, nur $1 / 4$ dieser Thoraxverletzungen treten isoliert auf. Meist sind sie somit aufgrund der Unfallmechanismen Teil einer Mehrfachverletzung, gelegentlich stellen sie eine schwere Einzelverletzung dar. Bauchtraumen sind zu 6\% beteiligt, hier ist etwa $1 / 3$ isoliert. Der Muskelmantel des Abdomens ist weniger stark ausgeprägt als bei Erwachsenen und die Oberbauchorgane sind weniger von den Rippen geschützt. Dadurch sind Leber, Milz, Nieren, Pankreas und auch der Darm leichter zu verletzen. Insgesamt stehen stumpfe Traumen ganz im Vordergrund, Schuss- oder Stichverletzungen sind in Mitteleuropa die Ausnahme. Das Verhältnis stumpfer zu scharfen Verletzungen liegt in Europa bei $9: 1$.

\section{Thoraxverletzungen}

\section{Epidemiologie und Verletzungsmuster}

Verkehrsunfälle und Stürze aus größerer Höhe stehen an 1. Stelle der Verletzungsursachen. Eine schwere Thoraxverletzung ist immer ein Hinweis auf die allgemeine Schwere des Unfalls und muss eine gründliche Suche nach anderen Traumafolgen veranlassen. Da die Rippen im Kindesalter viel flexibler sind, wird die auftreffende kinetische Energie direkt auf das Parenchym weitergeleitet. Rippenfrakturen treten deshalb nur in ca. 30\% auf. Lungenkontusionen sind mit 50\% die häufigeren Verletzungen, ein Pneumothorax tritt seltener auf als bei Erwachsenen. Die Elastizität der knöchernen Strukturen verhindert meist auch Sternumfrakturen sowie sternoklavikuläre Luxationen, sodass Schlüsselbeinfrakturen die einzigen häufigen Skelettverletzungen darstellen. Penetrierende Verletzungen von Lungen, Bronchien, Herz oder Gefäßen, Perikardergüsse und auch Zerreißungen von Trachea oder Ösophagus sind Ausnahmen [1].
Durch die Elastizität der Rippen ist bei geschlossener Stimmbandebene ein plötzlicher Druckanstieg im Brustkorb möglich. Es steigt der Druck in der Trachea und in der Lunge, dadurch wird ggf. eine Kompression der V. cava ausgelöst und es kommt zum Flüssigkeitsaustritt aus den Kapillaren und zu Mikroeinblutungen in Organe. Das kann Desorientiertheit und Bewusstseinsstörung, Zyanose, Einblutungen in die Bindehäute und Petechien sowie Krampfanfälle und eine Leberstauung auslösen.

\section{Merke}

Im Kindesalter kommt es am häufigsten zu Lungenkontusionen, ohne dass Rippenfrakturen auftreten und oft auch ohne einen Pneumothorax.

\section{Klinik}

Bei der Inspektion können Prellmarken auffallen, nach Verkehrsunfällen ist vor allem auf Gurtspuren zu achten. Eine inverse Brustkorbbeweglichkeit mit Rippeneinziehungen beim Einatmen kann ggf. am entkleideten Patienten festgestellt werden. Palpatorisch kann einerseits eine Instabilität des knöchernen Thoraxgerüstes imponieren, andererseits ist evtl. ein Hautemphysem zu tasten. Die dislozierte Klavikulafraktur ist ebenso zu palpieren wie eine hintere sternoklavikuläre Luxation (tastbare Einsenkung rechts oder links des oberen Brustbeines). Beim Abhören sind abgeschwächte Atemgeräusche Hinweis auf eine Lungenprellung, fehlende Atemgeräusche können auf einen Pneumothorax hindeuten. Beides kann jedoch durch schmerzhaft limitierte Atembewegungen verdeckt werden.

\section{Diagnostik}

Im Schockraum kann heute im Rahmen des erweiterten FAST (FAST = fokussierte abdominelle Sonographie bei Trauma) eine Ultraschalluntersuchung des Thorax erfolgen, die helfen kann, einen Pneumothorax, relevante Mengen freier Flüssigkeit oder einen Herzbeutelerguss früh zu erkennen. Häufig steht dann die konventionelle Thoraxröntgenaufnahme an 2. Stelle. Diese Untersuchung gibt immer noch eine gute Übersicht über die Thoraxverletzungen und erlaubt eine erste umfassendere Orientierung über das zu erwartende Verletzungsspektrum. Ohne Zweifel ist - mit Ausnahme von Leichtverletzten mit offensichtlich ausschließlich Bagatellverletzungen - in der Folge eine Computertomografie der Goldstandard, um das Ausmaß der Lungenverletzung ein- 
schätzen und - sofern der Patient noch spontan atmet die Notwendigkeit einer Beatmungstherapie voraussagen zu können. Die Kontrastmittelgabe lässt dabei auch die seltenen Gefäßverletzungen erkennen. Endoskopische Untersuchungen von Ösophagus und/oder Luftwegen sind im Kindesalter in der Akutphase selten indiziert. Sie bleiben meist der Abklärung relevanter und anhaltender freier thorakaler Luft nach Drainageanlage vorbehalten.

Merke

Nach eFAST und konventioneller Thoraxübersichtsaufnahme stellt das thorakale CT den Goldstandard zur Einschätzung thorakaler Verletzungen dar.

\section{Therapie}

Isolierte Thoraxprellungen sind die häufigste Diagnose bei isolierten Thoraxtraumen im Kindesalter. Therapeutisch steht die Analgesie im Vordergrund, eine antibiotische Prophylaxe verhindert die pneumonische Infiltration der eingebluteten Lungenabschnitte und die Physiotherapie leitet die Patienten zur adäquaten Respiration an. Bei schwereren Kontusionen und grenzwertig kompensierter Respiration empfiehlt es sich, nicht zu spät mit der Beatmung zu beginnen. Die Lunge benötigt bei Kindern etwas länger zur ausreichenden Erholung, weshalb besonders darauf geachtet werden muss, dass in der Folge nicht z. B. schon am Folgetag bei wiederhergestelltem Gasaustausch die Extubation veranlasst wird. Bei zu frühzeitiger Spontanatmung kann es zu einem Rebound mit erheblicher Verschlechterung der gesamten Atemsituation kommen. Das Kind mit einer beatmungsbedürftigen Lungenkontusion bedarf folglich eher 2 als einen Tag der mechanischen Atemhilfe.

Der Pneumothorax bedarf selbstverständlich einer adäquaten Drainage, die beim Kind nicht mittels Minithorakotomie angelegt werden muss. Nach initialer Entlastung über eine großlumige Punktionskanüle wird die Drainage auf gleichem Weg z. B. im 5. ICR in der mittleren Axillarlinie in Seldinger-Technik platziert.

Operative Eingriffe stellen die Ausnahme dar. Hier sind an 1. Stelle anhaltende Blutungen aus einer der seltenen Schuss- oder Stichwunden zu nennen, im Einzelfall auch einmal ein Leck in einem großen Bronchus, das sich nicht spontan schließt. Lebensbedrohliche Blutungen, die eine Clamshell-Thorakotomie (quere beidseitige Brustkorberöffnung inkl. Sternumdurchtrennung im $\sim 5$. ICR) erfordern, werden - wenn überhaupt - im Rahmen von Polytraumen beobachtet. Durch konsequente Physiotherapie und Mobilisation ggf. auch im Intensivbett muss die Entwicklung eines posttraumatischen Pleuraempyems, das dann einer thorakoskopischen Revision bedürfte, verhindert werden.

Osteosynthetische Maßnahmen stellen im Kindesalter eine Rarität dar. Die Klavikulafraktur bedarf nur bei dro- hender Perforation der Haut einer Stabilisierung, hierzu wird i.d.R. eine elastisch stabile Nagelung oder aber bei Jugendlichen eine Plattenosteosynthese gewählt. Rippen- und Sternumfrakturen von versorgungsrelevantem Ausmaß sind eine Rarität. Lediglich die hintere sternoklavikuläre Luxation (meist im Sinne einer medialen Epiphyseolyse der Klavikula), bei der das laterale Fragment Richtung Mediastinum zeigt, bedarf einer Reposition. Eine Fixation ist aber nicht immer nötig, ggf. „rastet“ die Klavikula ein und ist stabil, anderenfalls kann eine resorbierbare, transossäre Cerclage z. B. mit PDS-Kordel erfolgen.

Merke

Bei relevanter Lungenkontusion und erforderlicher Beatmung ist die zu frühe Extubation im Kindesalter zu vermeiden, um einen Rebound zu umgehen. Thoraxdrainagen können beim Kind i.d. R. ohne Minithorakotomie gelegt werden.

\section{Verläufe}

Relevante Folgeerscheinungen thorakaler Verletzungen sind im Kindesalter kaum bekannt. Das Lungenparenchym heilt und regeneriert schnell, sodass keine respiratorischen Einschränkungen verbleiben. Schwartenbildung ist die Ausnahme. Das Thoraxskelett remodelliert dem Alter entsprechend. Ösophageale oder bronchiale Verletzungen heilen schnell spontan ab oder sind durch die Regenerationskapazität des Gewebes auch nach einer Operation schnell wieder funktionell unbeeinträchtigt. Isolierte Thoraxtraumen sind somit in aller Regel kein Grund einer Invalidität im Kindesalter, sofern sie primär erkannt, konsequent diagnostiziert und therapiert und rehabilitiert werden. Beim Polytrauma wird der Verlauf in aller Regel vom Schädel-Hirn-Trauma bestimmt, hier wird der Langzeitverlauf aber ggf. durch initialen schweren Sauerstoffmangel aufgrund einer respiratorischen Insuffizienz negativ belastet.

\section{Abdominalverletzungen}

\section{Epidemiologie und Verletzungsmuster}

Abdominelle Verletzungen kommen im Kindesalter etwas häufiger vor als Thoraxverletzungen. Hinzu kommen typische Unfallmechanismen im Kindesalter wie Stürze auf den Fahrradlenker. Daneben muss auch bei Bauchtraumata an Verkehrsunfälle verschiedenster Konstellation und an Stürze aus größerer Höhe, aber auch an körperliche Auseinandersetzungen gedacht werden [2]. Für Kinder im Rahmen von Verkehrsunfällen ist bekannt, dass altersadäquate Rückhaltesysteme im Kraftfahrzeug das Verletzungsmuster positiv beeinflussen können, während inadäquate Sicherung zu einer deutlichen Zunahme vor allem von Hohlorganverletzungen führt. Isolierte Beckengurte können eine typische Trias mit LWS-Fraktur, Pankreasverletzung und Dünndarmtrauma hervorrufen. 
- Tab. 1 OIS (organ injury scale) der AAST (American Association of Surgery in Trauma).

\begin{tabular}{|c|c|c|c|}
\hline & Niere & Milz & Leber \\
\hline 1 & subkapsuläres Hämatom & $\begin{array}{l}\text { - subkapsuläres Hämatom < } 10 \% \\
\text { - Lazeration }<1 \mathrm{~cm} \text { tief }\end{array}$ & $\begin{array}{l}\text { - subkapsuläres Hämatom < 10\% } \\
\text { - Lazeration < } 1 \mathrm{~cm} \text { tief }\end{array}$ \\
\hline ॥ & Lazeration $<1 \mathrm{~cm}$ & $\begin{array}{l}\text { - subkapsuläres Hämatom < } 50 \% \\
\text { - Lazeration }<3 \mathrm{~cm} \text { tief }\end{array}$ & $\begin{array}{l}\text { - subkapsuläres Hämatom < } 50 \% \\
\text { - Lazeration }<3 \mathrm{~cm} \text { tief }\end{array}$ \\
\hline III & Lazeration $>1 \mathrm{~cm}$ & $\begin{array}{l}\text { - subkapsuläres Hämatom >50\% } \\
\text { - Lazeration >3cm tief }\end{array}$ & $\begin{array}{l}\text { - subkapsuläres Hämatom > 50\% } \\
\text { - Lazeration >3 cm tief }\end{array}$ \\
\hline IV & $\begin{array}{l}\text { - ausgedehnte Lazeration } \\
\text { - Kelchbeteiligung }\end{array}$ & $\begin{array}{l}\text { " Ruptur } \\
\text { - segmentale Destruktion > 25\% }\end{array}$ & $\begin{array}{l}\text { - Ruptur } \\
\text { - Lazeration bis } 50 \% \text { eines Leberlappens }\end{array}$ \\
\hline V & $\begin{array}{l}\text { - Zertrümmerung } \\
\text { - vaskuläre Läsion }\end{array}$ & vollständige Destruktion & $\begin{array}{l}\text { - Lazeration > 50\% eines Leberlappens } \\
\text { - Kavaverletzung } \\
\text { - Lebervenenverletzung }\end{array}$ \\
\hline $\mathrm{VI}$ & & & Zerreißung \\
\hline
\end{tabular}

Polytraumen sind im Kindesalter selten. Dennoch sind viele Erstversorgungsalgorithmen darauf abgestellt. Es ist auch durchaus nicht falsch, Kinder nach einem adäquaten Unfallmechanismus bis zum Beweis des Gegenteils nach den Kriterien der Polytraumaversorgung zu behandeln, auch wenn es sich am Ende der Diagnostik nur als Monotrauma oder als schwere Mehrorganverletzung ohne Lebensgefahr (und damit per definitionem nicht als Polytrauma) herausstellt.

Dabei steht die Milzverletzung vor der Leber und der Niere an 1. Stelle. Pankreas, Duodenum und übriger Gastrointestinaltrakt sind deutlich seltener betroffen. Unterschätzt wird oft der Anteil der Nierenverletzungen im Kindesalter, da diese - wenn sie nicht kinderchirurgisch, sondern urologisch versorgt werden - in den Statistiken oft nicht adäquat aufgeführt werden. Gerade bei den Nieren führt die geringere Schutzfunktion der Rippen zu einer größeren Verletzungshäufigkeit bei den jüngeren Patienten.

\section{Merke}

Milz, Leber und Niere sind die am häufigsten verletzten Organe. Altersadäquate Rückhaltesysteme im Auto verhindern vor allem Hohlorganverletzungen.

\section{Klinik}

Bei der Inspektion ist zu allererst auf Kontusionsmarken (z.B. Fahrradlenker) und Gurtzeichen zu achten. Bei der Palpation können Druckschmerz, Klopfschmerz (vor allem Nierenlager) und Abwehrspannung registriert werden. Auskultatorisch weisen ggf. fehlende Darmgeräusche auf eine lleussituation im Sinne einer reflektorischen Paralyse hin. Auf Blutdruck und Herzfrequenz ist zu achten, um Anzeichen eines relevanten Volumenverlustes nicht zu übersehen.

\section{Diagnostik}

Auch bei der abdominellen Monoverletzung gehört FAST heute zu den Schockraumalgorithmen. Findet sich freie Flüssigkeit oder ergibt sich hier der Hinweis auf eine Organverletzung, kann bei stabilen Kreislaufverhältnissen noch einmal fokussiert im Ultraschall nach der Art und dem Ausmaß der Organläsion gesucht werden. Dabei erreicht die Ultraschalluntersuchung in erfahrener Hand durchaus gute Sensibilität und Spezifität von 90-95\%, verstärkt unter der Anwendung von Kontrastmittel. Freie Flüssigkeitsmenge und Verletzungsausmaß sind relativ gut abzuschätzen. Nicht jede isolierte Organverletzung bedarf einer Computertomografie, auch wenn diese heute als der Goldstandard gilt und eine Sensibilität/Spezifität von $98 \%$ erreicht. Wenn jedoch therapierelevante Zusatzinformationen zu erwarten sind, darf mit der Indikation zur CT nicht gezögert werden. Auch wenn verschiedene Betrachter bei der Beurteilung einer $\mathrm{CT}$ nicht absolut übereinstimmen, kann das Ausmaß der Organverletzung hier am korrektesten eingeschätzt werden. Entsprechend basiert die Klassifikation der Organverletzungen nach der American Society of Surgeons auf der CT und unterscheidet 4-5 Typen pro Organ ( $\triangleright$ Tab. 1). Bei unauffälliger CT und stabilem Kind wird in den angloamerikanischen Ländern die Entlassung propagiert [3].

Als „Nebenbefund“ kann die CT Wandhämatome im Duodenum oder aber freie Luft als Hinweis auf eine Magen-Darm-Perforation beschreiben. Falls die Perforation in dieser frühen Phase aber noch gar nicht vorliegt, weil der betroffene Darmabschnitt noch dicht ist, kann bei entsprechender Klinik im Verlauf auch die konventionelle Abdomenübersichtsaufnahme einen Perforationsverdacht klären helfen (Abdomen a.-p. und in Linksseitenlage).

Parallel erfolgt die Analyse der Laborwerte mit Blutbild, Gerinnung und den Serumwerten für Niere, Leber und Pankreas. Erhöhte Transaminasen haben eine hohe Sensibilität für eine Leberverletzung und sind auch für die Ver- 
laufskontrolle gut geeignet. Ähnliches gilt für die Pankreaswerte nach Pankreasverletzung.

Radiologisch-interventionelle Verfahren stehen zwischen Diagnostik und Therapie [4] und werden beim Management der einzelnen Organläsionen aufgeführt.

\section{Merke}

In der Diagnostik der abdominellen Organverletzungen folgt auf das initiale FAST die CT vor allem zum Grading, ggf. später ergänzt durch eine konventionelle Übersichtsaufnahme, wenn es lediglich um eine sekundäre Perforation im Magen-Darm-Trakt geht. Laborwerte sind sensibel bei Leber- und Pankreastraumen.

\section{Therapieprinzipien}

Das Management stumpfer Organverletzungen im Kindesalter ist heute sehr stark konservativ geprägt. Es gibt gute und evidente Daten, die dieses Vorgehen unterstützen. Dadurch hat auch die Laparoskopie in diesem Bereich keine große Bedeutung erhalten [5]. Diagnostische Bauchspiegelungen führen nur selten zu einer klareren Einschätzung als die $\mathrm{CT}$, interventionelle Optionen beschränken sich auf den Verschluss einzelner klar identifizierbarer gastrointestinaler Perforationen oder von Zwerchfellverletzungen [6]. Die Applikation hämostatischer Vliese auf blutende Parenchymwunden kann bei einer signifikanten Blutung sicher nicht zum Erfolg führen und ist bei einer geringer ausgeprägten Blutung entbehrlich. Die heute verbreitete Erfahrung mit der Laparoskopie als Methode sollte nicht dazu führen, abdominelle Blutungen zu inspizieren, deren konservative Behandlungsmöglichkeiten bewiesen sind, und damit das akute Traumamanagement durch eine Operation ohne zwingende Indikation zu komplizieren.

\section{Milzverletzungen}

Bei den Milzverletzungen werden 5 Verletzungsausprägungen unterschieden ( $\triangleright$ Tab. 1). Im Ultraschall finden sich direkte Zeichen der Organverletzung, daneben freie Flüssigkeit im Oberbauch und im kleinen Becken. Freie Flüssigkeit ist keine OP-Indikation! Die CT klassifiziert das Verletzungsausmaß. Mit Ausnahme der Typ-V-Verletzung ist die Behandlung primär konservativ. Dieser auf die Initiativen des Childrens Hospital of Toronto in den Jahren seit 1955 zurückgehende Ansatz hat sich zwischenzeitlich weltweit etabliert. In Toronto wurden zuletzt konservative Vorgehensweisen in bis zu $97 \%$ unter Einschluss der polytraumatisierten Kinder praktiziert, die Quote des Organverlustes lag bei 1\%, die Häufigkeit von Transfusionen ebenfalls und die Mortalität isolierter Milzverletzungen war von 8 auf $0,7 \%$ gesunken [7]. Stylianos und Mitarbeiter haben für die isolierte Milz- (und Leber-)Verletzung einen evidenzbasierten Algorithmus veröffentlicht, der - unter den ganz anderen Grundvoraussetzungen des US-amerikanischen Gesundheitssystems - auch die
- Tab. 2 Intensivüberwachung und Hospitalisation, Bildgebung vor/nach der Entlassung und Beschränkung der sportlichen Aktivität entsprechend dem Algorithmus von Stylianos (2002).

\begin{tabular}{|l|c|c|c|c|}
\hline CT-Grad & I & II & III & IV \\
\hline Intensiv-Tage & - & - & - & 1 \\
\hline Krankenhaus-Tage & 2 & 3 & 4 & 5 \\
\hline Bildgebung vor/nach Entlassung & - & - & - & - \\
\hline Einschränkung Aktivität/Wochen & 3 & 4 & 5 & 6 \\
\hline
\end{tabular}

Dauer der intensivmedizinischen und der allgemeinen stationären Überwachung, die Häufigkeit der bildgebenden Nachkontrollen und den Umfang der Sportkarenz vorschlägt und als Orientierung dienen kann [8, 9] (॰ Tab. 2). Grundvoraussetzung ist für dieses konservative Management die optimale Bildgebung, die pädiatrisch-intensivmedizinische Überwachung und darunter die Gewährleistung der Kreislaufstabilität. Wenn der Blutdruck - durchaus auch unter Katecholamingabe und Volumensubstitution - stabil bleibt und die erforderliche Transfusionsmenge hierzu $40 \mathrm{ml} / \mathrm{kg}$ Körpergewicht in 24 Stunden nicht überschreitet, kann das konservative Vorgehen beibehalten werden. Interessant ist, dass unter diesem Regime sowohl die Menge des transfundierten Blutes als auch die Komplikationsrate signifikant sinken.

Die interventionelle Embolisierung blutender Milzgefäße hat sich im vergangenen Jahrzehnt auch für das Kindesalter als praktikabel erwiesen und ermöglicht bei anhaltender profuser Blutung die Vermeidung der Laparotomie. Gelegentlich tritt im Verlauf an der Milzarterie ein Pseudoaneurysma auf, das ein sekundäres Blutungsrisiko mit sich bringt. Auch hier kann interventionell vorgegangen werden $[4,10]$.

Typ-V-Verletzungen mit Abriss des Milzstiels bedürfen ohne Zweifel der notfallmäßigen Laparotomie zur Versorgung der Arterie und der Bergung des Organs [11]. Hier ist im Verlauf wegen des ungewöhnlichen Sepsisrisikos (OPSI-Syndrom = overwhelming post-splenectomy infection) an die Pneumokokken- und Hämophilusimpfung und eine langfristige Antibiotikaprophylaxe entsprechend den Empfehlungen der DGPI (Deutsche Gesellschaft für pädiatrische Infektiologie) zu denken.

Die zweizeitige Kapselruptur stellt im Kindesalter eine absolute Rarität dar, was ggf. auf die festere Kapselstruktur des Kindes zurückgeführt werden kann. Dadurch sind im Verlauf kaum Probleme zu erwarten. Weder muss das Kind auf der Station wochenlang Bettruhe einhalten, noch sind nach Entlassung monatelange Sportkarenzen indiziert. Sechs Wochen nach dem Trauma hat sich die Milzverletzung konsolidiert und der Patient kann wieder seinem normalen Alltagsleben nachgehen. 
Merke

Freie Flüssigkeit ist keine OP-Indikation. Entscheidend ist die Kreislaufstabilität und die Menge an substituierten Blutprodukten (Grenze: 40 ml/kg Körpergewicht/24 h etwa halbes Blutvolumen). Konservative Therapie steht ganz im Vordergrund. Interventionelle Embolisierungen sind ggf. möglich. Die zweizeitige Ruptur ist im Kindesalter eine Rarität.

\section{Leberverletzungen}

Die Einteilung der Leberverletzungen ist ähnlich derjenigen der Milzverletzungen. Auch bei der Leber hat sich zunehmend das konservative Vorgehen durchgesetzt, auch wenn der Anteil konservativ behandelter Lebertraumen noch nicht die Quote wie bei den Milzverletzungen erreicht hat. Auf jeden Fall gilt heute auch bei klinischen (Gurt- oder Fahrradlenkermarke im rechten Oberbauch) oder sonografischen (freie Flüssigkeit parahepatisch, direkte hepatische Verletzungszeichen) Zeichen des Lebertraumas zunächst das konservative Vorgehen. Die von Stylianos aufgestellten Kriterien sind auch für die isolierte Leberverletzung bewiesenermaßen wirksam und die Kriterien der Kreislaufstabilität gelten in gleicher Weise. Zudem lässt sich die Leberverletzung sehr gut an den Transaminasen im Serum überwachen, die schnell bei der Parenchymverletzung ansteigen, sich aber auch rasch wieder normalisieren.

In Einzelfällen sind Segmentabrisse mit Eröffnung größerer Gefäße möglich, die bei der Leber doch etwas häufiger eine Operation erfordern [11]. In Kenntnis der Techniken segmentaler Leberresektionen kann hier entsprechend vorgegangen werden.

Zwei Besonderheiten sind bei der Leberverletzung zu bedenken: begleitende Verletzungen der Gallengänge sind zu beachten und an die mögliche retrohepatische Kavaverletzung ist zu denken. Für ein Gallenleck gilt zunächst auch ein konservatives Management mit Drainage, ggf. aber auch mit endoskopischer Einlage eines Stents, um die Verletzung zur Abheilung zu bringen. Nur in Ausnahmefällen wird sich hier die Indikation zu einer operativen Revision ergeben. Dagegen erfordert die Verletzung der Hohlvene im Abschnitt hinter der Leber große interventionelle oder operative Erfahrung, sodass hier - sofern möglich - die Hinzuziehung von oder Verlegung zu erfahrenen Leberoperateuren in Betracht gezogen werden muss. Sollte jedoch eine Leberblutung intraoperativ nicht zu beherrschen sein, muss ein sog. Packing mit Bauchtüchern erfolgen, um das Verbluten zu verhindern. Mit der Kompression der Blutungsquelle lässt sich Zeit gewinnen, um Unterstützung zu organisieren oder den Kreislauf auf der Intensivstation zu stabilisieren.

Für die Verläufe isolierter Leberverletzungen gelten die gleichen von Stylianos aufgestellten Rehabilitationszeiträume wie bei der Milz.
Merke

Konservatives Management unter Monitoring von Kreislauf und Transaminasen steht im Vordergrund. Bei schweren Blutungen ist an das Packing und die Hinzuziehung entsprechender Expertise zu denken.

\section{Nierenverletzungen}

Wegen der geringeren Bedeckung der Nieren im Kindesalter durch die Rippen und die weniger ausgeprägte Bauchmuskulatur sind renale Verletzungen im Kindesalter häufiger als bei Erwachsenen anzutreffen. Die Klassifikation entspricht annähernd derjenigen der Milzverletzungen. Als Besonderheit kommt die mögliche traumatische Eröffnung des Nierenbeckens hinzu. Deshalb kann es sich bei freier Flüssigkeit primär immer sowohl um Blut als auch um Urin handeln. Kurzfristig wird sich zeigen, ob es zur Koagelbildung oder aber zur Zunahme eines Urinoms kommt. Bei der Blutung gilt zunächst wie bei Milz und Leber die Organerhaltung als oberstes Ziel, und die Vermeidung einer operativen Intervention ist in aller Regel möglich [12 - 14]. Interventionelle Drainagen oder Ableitungen mittels Doppel-J-Katheter können die Chance zur konservativen Therapie auch bei Verletzungen der ableitenden Harnwege aufrechterhalten. Bei posttraumatischen Durchblutungsstörungen, z. B. durch eine Intimaläsion, besteht durch die angiografische Intervention die Möglichkeit zum Organerhalt.

Nur bei Nierenverletzungen Grad IV oder V oder einem vollständigen Nierenstielabriss stehen eine operative Intervention mit dem Versuch der Rekonstruktion oder aber eine notfallmäßige Nephrektomie zur Debatte. Die Quote liegt bei $10-15 \%$ und ist weiter rückläufig. In einigen Fällen sind Spätnephrektomien nach Funktionsverlust und bei Entwicklung von Bluthochdruck indiziert.

Nach Resorption von Hämatom und/oder Urinom und zur Heilung der Nierenbeckenwunde wird der Doppel-JKatheter für 6-8 Wochen belassen, bevor er ambulant entfernt werden kann. Die Nierenfunktion kann nach 6 Monaten einmal szintigrafisch überprüft werden, im Vordergrund steht aber die sonografische Kontrolle bez. Organgröße, -struktur und -dichte sowie die Blutdrucküberwachung. Langzeitkontrollen sind nicht erforderlich, wenn eine komplikationslose Abheilung der Organläsion ohne relativen Funktionsverlust eingetreten ist.

\footnotetext{
Merke

Freie Flüssigkeit kann Blutung oder Urinom sein. Interventionelle Drainage oder Doppel-J-Einlage gehören hier zum konservativen Management. Die Quote des Organverlustes - ggf. auch im Langzeitverlauf liegt bei $15 \%$.
} 


\section{Pankreasverletzungen}

Pankreasverletzungen galten lange als die Organtraumata, die noch der chirurgischen Exploration, der gezielten Drainage, ggf. der Gang- oder Organnaht oder sogar der Teilresektion bedurften. Es hat sich jedoch auch hier in den letzten Jahren eine deutliche Hinwendung zum konservativen Vorgehen entwickelt, mehr als die Hälfte der Fälle wird ohne Operation behandelt [15]. Die interventionell eingelegte Abdominaldrainage oder aber der Stent via ERCP (ERCP = endoskopische retrograde Cholangio-Pankreatikografie) zur Schienung des verletzten Ductus wirsungianus haben die operativen Maßnahmen zurückgedrängt, basieren aber auf einer guten Bildgebung mit CT und/oder MRT. Dabei sind die Komplikationen durchaus noch hoch und realisieren sich bei konservativem Management eher am Pankreas selbst (Pseudozyste), nach Operation eher extrapankreatisch (Duodenum, Milz).

Viele Pankreasverletzungen bedürfen jedoch keiner Operation. Mit der Bestimmung der Amylase und der Lipase im Serum stehen spezifische Laborparameter zur Überwachung der Verletzungsaktivität zur Verfügung. Unter Magensaftblockade und -drainage, passagerer Nahrungskarenz und vorsichtigem Nahrungsaufbau ist häufig eine gute Rehabilitation der Verletzten möglich. Zu bedenken ist, dass bei Pankreasverletzungen am häufigsten Begleitverletzungen an Hohlorganen zu finden sind.

Wurde ein Stent in den Pankreasgang eingelegt, so muss dieser nach einigen Wochen entfernt werden. Dies gibt ggf. noch einmal die Möglichkeit, den Gang darzustellen, seine Suffizienz und Durchgängigkeit zu prüfen und den regulären Abfluss zu kontrollieren.

Auch die Entwicklung einer posttraumatischen Pankreaspseudozyste führt heute eher zur Intervention einschließlich einer endoskopischen Gastrozystostomie [16]. Nur bei anhaltendem Austritt von Pankreassekret in die freie Bauchhöhle mit resultierenden Zeichen der floriden Pankreatitis und Peritonitis kann die Revision erforderlich werden. Dabei gilt natürlich auch hier primär der Organerhalt, was ggf. eine Pankreasableitung im Sinne einer Zystojejunostomie oder aber eine Pankreasschwanzresektion mit/ohne Ableitung in eine ausgeschaltete Jejunumschlinge erfordert. Eine solche Entwicklung einer posttraumatischen Pankreaspseudozyste kann durchaus erst Monate später auftreten und wird meist durch unspezifische Oberbauchbeschwerden bemerkt. Ihre Entstehung hängt nicht allein von der Schwere der Verletzung $a b$ und kann durchaus auch nach leichteren Pankreastraumen auftreten. Deshalb ist nach Pankreasverletzungen eine längere klinische, sonografische und laborchemische Nachsorge bis 1 Jahr post Trauma zu empfehlen. Eine langfristige Diät ist dagegen heute nicht mehr empfohlen, diese beschränkt sich auf die ersten Tage nach der Verletzung.
Merke

Pankreasverletzungen sind häufiger als andere Organtraumen mit Komplikationen behaftet und gehen oft mit gastrointestinalen Verletzungen einher. Interventionelle Maßnahmen (Stent, Drainage) kommen vor einer Resektion oder Ableitung. Wegen der möglichen Entwicklung von Pseudozysten bedarf es längerer Nachkontrollen.

\section{Verletzungen des Gastrointestinaltraktes}

Als noch jeder sonografische freie Flüssigkeitsnachweis zur Laparotomie führte, wurden häufig im Nebenschluss bei der Durchsicht des Magen-Darm-Traktes auch gastrointestinale Kontusionen oder Perforationen diagnostiziert und ggf. versorgt. Im Zeitalter der konservativen Behandlungsstrategien entfällt dieser Automatismus. Deshalb muss den Verletzungen von Magen und Darm mehr Augenmerk geschenkt werden. Von den Unfallmechanismen sprechen vor allem Einschnürungen durch schlechtsitzende Beckengurte für ein Trauma des Verdauungstraktes [17]. Das obstruktive Wandhämatom im Duodenum heilt meist unter Magensondenentlastung spontan ab. Die freie Perforation erfordert zweifelsfrei eine Intervention. Hier kommt bei entsprechend übersichtlicher Gesamtkonstellation durchaus die Laparoskopie infrage, um die Perforationsstelle zu identifizieren und ggf. auch zu versorgen. Kann hier eine Laparotomie vermieden werden, reduziert das die Belastung des Patienten. Anderenfalls ist die Perforation oder Zerreißung offen zu versorgen. Dabei erfordert die isolierte gastrointestinale Verletzung sicher nur in absoluten Ausnahmefällen die Anlage eines Anus praeter, sondern erlaubt in aller Regel die direkte Naht unter anschließendem adäquatem Nahrungsaufbau. Bei lokalisiertem Geschehen ist eine Drainage vielleicht entbehrlich, aber selten schädlich. Interessant ist, dass nach neueren Daten die Regel, dass je später die Versorgung erfolgt, desto größer das Komplikationspotenzial sei, nicht mehr stimmt. Es konnte gezeigt werden, dass auch nach verzögerter Versorgung einer gastrointestinalen Perforation der Verlauf günstig war [18].

Gastrointestinale Verletzungen fallen heute häufiger als andere Organtraumen sekundär auf. Während wie bereits ausgeführt sekundäre Milzrupturen entgegen der jahrzehntelangen Überzeugung zumindest im Kindesalter kaum auftreten, muss die mittelfristig anhaltende peritoneale Symptomatik den Verdacht auf eine übersehende oder initial nicht symptomatische Darmverletzung lenken und im Verlauf noch einmal eine Bildgebung indizieren.

\footnotetext{
Merke

Gastrointestinale Verletzungen werden häufiger sekundär diagnostiziert. Die verzögerte operative Versorgung erhöht bei Kindern nicht die Komplikationsrate. Ein Anus praeter ist nur in Ausnahmefällen erforderlich.
} 


\section{Zusammenfassung}

Organverletzungen sind im Kindesalter sowohl im Thorax als auch im Abdomen selten. Eine gute Bildgebung durch Ultraschall und/oder CT ist die Basis einer Gradeinteilung, mit der sich dann die Therapie ableiten lässt. Solange der Kreislauf stabil ist und keine Peritonitis vorliegt, steht die konservative Behandlung grundsätzlich im Vordergrund. Interventionelle Möglichkeiten sind auf dem Vormarsch. Das darf in der Summe nicht dazu führen, dass die Prinzipien der chirurgischen Versorgung nicht mehr präsent sind. Zumindest in den kindertraumatologischen Referenzzentren der Traumanetzwerke müssen von Packing über Organnaht und Segmentresektion bis zu Rekonstruktion die entsprechenden Techniken beherrscht werden. Andererseits garantiert nach internationaler Literatur die kinderintensivmedizinische Expertise häufiger die Umsetzung der konservativen Algorithmen und reduziert z. B. signifikant die posttraumatische Splenektomie.

Interessenkonflikt

Der Autor erklärt, dass kein Interessenkonflikt vorliegt.

\section{Autorinnen/Autoren}

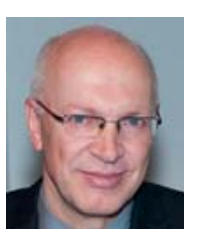

\section{Peter P. Schmittenbecher}

Prof. Dr., Chefarzt, Kinderchirurgische Klinik, Städtisches Klinikum Karlsruhe

Korrespondenzadresse

Prof. Dr. Peter P. Schmittenbecher

Kinderchirurgische Klinik

Städtisches Klinikum

Moltkestraße 90

76133 Karlsruhe

Tel.: 0721/974-3101

peter.schmittenbecher@klinikum-karlsruhe.de

\section{Literatur}

[1] Fitze G. Das Thoraxtrauma im Kindesalter. Kinder- und Jugendmedizin 2007; 7: 73-78

[2] Wessel LM. Das stumpfe Bauchtrauma im Kindesalter. Trauma Berufskrankh 2014; 16 (Suppl. 3): S311-S316

[3] Braungart S, Beattie T, Midgley P et al. Implications of a negative abdominal CT in the management of pediatric blunt abdominal trauma. J Pediatr Surg 2017; 52: 293-298
[4] Kiankhooy A, Sartorelli KH, Vane DW et al. Angiographic embolization is safe and effective therapy for blunt abdominal solid organ injury in children. J Trauma 2010; 68: 526-531

[5] Gaines BA, Rutkoski JD. The role of laparoscopy in pediatric trauma. Sem Pediatr Surg 2010; 19: 300-303

[6] Marvan A, Harmon CM, Georgeson KE et al. Use of laparoscopy in the management of pediatric abdominal trauma. J Trauma 2010; 69: 761-764

[7] Davies DA, Pearl RH, Ein SH et al. Management of blunt splenic injury in children: evolution of the nonoperative approach. J Pediatr Surg 2009; 44: 1005-1008

[8] Stylianos S. Evidence-based guidelines for resource utilization in children with isolated spleen or liver injury: the APSA Trauma Committee. J Pediatr Surg 2000; 35: 164-169

[9] Stylianos S. Compliance with evidence-based guidelines in children with isolated spleen or liver injury: a prospective study. J Pediatr Surg 2002; 37: 453-456

[10] Safavi A, Beaudry P, Jamieson D et al. Traumatic pseudoaneurysms of the liver and spleen in children: is routine screening warranted? J Pediatr Surg 2011; 46: 938-941

[11] Fakhry T, Sabry A, Shawky M. Factors for failure of nonoperative management of blunt hepatosplenic trauma in children. Ann Pediatr Surg 2016; 12: 63-67

[12] Umbreit EC, Routh JC, Husmann DA. Nonoperative management of nonvascular grad IV blunt renal trauma in children: meta-analysis and systematic review. Urology 2009; 74: 579582

[13] Fitzgerald CL, Tran P, Burnell J et al. Instituting a conservative management protocol for pediatric blunt renal trauma: evaluation of a prospective maintained patient registry. J Urol 2011; 185: 1058-1064

[14] Okur MH, Arslan S, Aydogdu B et al. Management of highgrade renal injury in children. Eur ] Trauma Emerg Surg 2017; 43: 99-104

[15] Englum BR, Gulack BC, Rice HE et al. Management of blunt pancretic trauma in children: Review of the National Trauma Data Bank. J Pediatr Surg 2016; 51: 1526-1531

[16] Blaauw ID, Winkelhorst JT, Rieu PN et al. Pancreatic injury in children: good outcome of nonoperative treatment. J Pediatr Surg 2008; 43: 1640-1643

[17] Paris C, Brindamour M, Ouimet A et al. Predictive indicators for bowel injury in pediatric patients who present with a positive seat belt sign after motor vehicle collision. J Pediatr Surg 2010; 45: 921-924

[18] Letton RW, Worrell V, APSA Committee on Trauma Blunt Intestinal Injury Study Group. Delay in diagnosis and treatment of blunt intestinal injury does not adversely affect prognosis in the pediatric trauma patient. J Pediatr Surg 2010; 45: 161-166

Bibliografie

DOI https://doi.org/10.1055/a-0624-5033

OP-JOURNAL 2018; 34: 254-260 @ Georg Thieme Verlag KG Stuttgart · New York ISSN 0178-1715 